\title{
Morality, Normativity, AND THE GoOd System 2 FALlacy
}

\author{
- Wim De Neys -
}

\begin{abstract}
In this commentary, I warn against a possible dual process misconception that might lead people to conclude that utilitarian judgments are normatively correct. I clarify how the misconception builds on (1) the association between System 2 and normativity in the dual process literature on logical/probabilistic reasoning, and (2) the classification of utilitarian judgments as resulting from System 2 processing in the dual process model of moral reasoning. I present theoretical and empirical evidence against both premises.
\end{abstract}

Keywords: reasoning; decision making; dual process theory; moral reasoning; normative fallacy

Published online: 12 February 2020

\section{Introduction}

The papers in this special issue explore whether we can draw any normative conclusions by discovering the psychological processes underlying moral judgments. In my opinion, the short answer to this question is a resounding "No!". But instead of bothering readers with my amateur philosophical musings about why we cannot move from "is-to-ought" I will focus on warning against a lingering misconception - fuelled by popular dual process research - that might distort some readers' conclusions.

In general, the dual process model conceives of human thinking as an interplay of fast intuitive and slower, more deliberate processing. Often, these two types of thinking are referred to as System 1 and System 2 thinking, respectively. ${ }^{1}$ The model initially gained popularity in the 1970s because it presented an appealing explanation for observed biases in people's logical and probabilistic reasoning. ${ }^{2}$ This literature suggested that biases in classic reasoning tasks were often associated with the operation of the intuitive System 1, whereas correct responding in line with traditional logical and probabilistic norms was associated with the successful engagement of the deliberate System 2. As I will clarify in more detail below, this association has led to the errone-

\author{
Wim De Neys \\ LaPsyDE (UMR CNRS 8240) \\ Sorbonne - Labo Binet \\ Paris Descartes University \\ 46 Rue Saint-Jacques \\ 75005 Paris, France \\ e-mail: wim.de-neys@parisdescartes.fr \\ ${ }^{1}$ Kahneman (2011). \\ ${ }^{2}$ De Neys, Pennycook (2019); for an excellent historical account see Frankish, Evans (2009).
}


ous assumption that System 2 processing is by definition normatively correct (i.e., the normative fallacy) $)^{1}$.

In more recent years, the core dual process principles have been applied in a wide range of fields, including work on the psychological basis of moral reasoning. ${ }^{2}$ The dual process model of moral cognition posited that making deontological moral decisions in hard sacrificial dilemmas results from System 1 processing, whereas making utilitarian decisions was assumed to require System 2 processing ${ }^{3}$. Combining the normative fallacy and the dual process model of moral reasoning might give rise to the following "moral normativity" inference:

(1) System 2 thinking is normatively correct

(2) Utilitarian judgments result from System 2 thinking

(3) Therefore, utilitarian judgments are normatively correct

In other words, given (1) the association between System 2 and normativity in the dual process literature on logical/probabilistic reasoning, and (2) the classification of utilitarian judgments as resulting from System 2 processing in the moral reasoning field, one might be led to conclude that utilitarian judgments are normatively "correct" (3). In the present paper, I will present theoretical and empirical evidence against this misconception. I will start by briefly discussing the origin of the normative fallacy.

\section{Dual process models and reasoning biases}

Research on reasoning and decision making has shown that even educated people readily violate the most basic logical and probabilistic normative principles in a wide range of classic reasoning tasks. ${ }^{4}$ These reasoning tasks are constructed such that they cue a strong intuitive response that conflicts with the response that is considered correct in standard logic or probability theory. ${ }^{5}$ A good modern-day example of such a classic task is the bat-and-ball problem (i.e., "A bat and a ball cost $\$ 1.10$ in total. The bat costs $\$ 1$ more than the ball. How much does the ball cost?). When faced with this problem, the response " 10 cents" readily comes to mind. However, upon reflection, it is clear that 10 cents cannot be right (i.e., at a dollar more the bat would cost $\$ 1.10$ which gives a total of $\$ 1.20$ ) and the ball rather costs 5 cents.

The traditional dual process model presents an elegant explanation for the observed "bias" in our thinking. ${ }^{6}$ The early dual process theorists assumed that processing the basic logico-mathematical principles that allow arriving at the correct response

\footnotetext{
${ }^{1}$ Evans (2011).

2 E.g. Greene (2013); Greene, Haidt (2002).

${ }^{3}$ In sacrificial dilemmas (e.g., the infamous "Trolley" problem) people need to indicate whether they agree to sacrifice the life of one (some) individual(s) to save the lives of more individuals. Utilitarian decisions are those in which people opt to save the lives of more (e.g., Greene, 2013).

${ }^{4}$ Evans (2002); Kahneman (2011).

${ }^{5}$ Whether these norms are appropriate and ultimately correct is yet another discussion - see Stanovich, West (2000).

${ }^{6}$ Evans, Stanovich (2013); Kahneman (2011).
} 
typically requires deliberate, System 2 processing. However, because people tend to minimize demanding computations, they will refrain from engaging or completing the effortful System 2 processing whenever the intuitive System 1 cues a plausible answer. Hence, although people might, in theory, solve the classic reasoning task correctly by engaging in System 2 thinking, in practice they will often end up being biased because they rely on the less demanding, intuitively cued System 1 response.

\section{Normative fallacy}

The above section clarifies that within the context of classic logical and probabilistic reasoning tasks in which the dual process framework evolved, it was assumed that there will be an association between type of processing (System 1 or 2) and the normative status of the response. Within this specific context, one could claim that reasoners who successfully engage System 2 will be more likely to give the normatively correct response. ${ }^{7}$ The problem is that this specific association has been interpreted as a general feature of System 1 and System 2 processing. Under this interpretation System 1 activation is equated with error and System 2 activation with sound reasoning. Put bluntly, System 1 is perceived as "bad" and System 2 processing is "good".

It should be crystal clear that the early dual process theorists have long argued against this simplification and over-generalization. It is often referred to as the normative fallacy. ${ }^{8}$ Dual process proponents never claimed that intuitive processing necessarily leads to errors and deliberate processing necessarily leads to normatively correct responses. Deliberate processing was never believed to be some magical gateway to normativity.

Normative correctness is not a defining feature of System 2 processing. ${ }^{9}$ The mistake here is that people have taken the specific association between type of processing and normative correctness in the context of some classic reasoning tasks as a general feature of System 1 and 2 processing.

There is abundant evidence that clearly shows that intuitive processing can lead to correct problem solutions whereas deliberation can lead us astray. ${ }^{10}$ To give but one example, in one study Beilock and DeCaro presented participants with math-based questions such as "Does the answer to the problem (32-8) / 4 have a remainder?". ${ }^{11}$ One can solve this problem by relying on a deliberate strategy and work through various step-by-step computations (i.e., subtracting 8 from 32 and then dividing this answer by 4 so as to conclude that the answer is no). A simpler intuitive "short-cut" strategy" is to rely on the fact that all the numbers in the problem are even. Often (but not always), when subtracting and dividing even numbers there are no remainders. Studies showed that on tests in which the intuitive strategy resulted in a correct response, people who

\footnotetext{
${ }^{7}$ Again, the label "normative" refers here to the response that is traditionally considered to be correct in standard logic or probability theory, see footnote 7.

${ }^{8}$ Evans (2011).

${ }^{9}$ Evans, Stanovich (2013).

${ }^{10}$ For an overview e.g. Amer, Campbell, Hasher (2016).

11 Beilock, DeCaro (2007).

${ }^{12}$ I readily admit that one can argue about how "intuitive" this strategy really is. The point is that it is presumably less demanding than the alternative strategy.
} 
score lower on cognitive capacity tests (i.e., people who will be less likely to engage in deliberate processing $)^{13}$ outperformed the cognitively more gifted participants. Higher capacity reasoners were more likely to use the more complex strategy and were at risk of making calculation errors when doing so.

The general point I am trying to make is that knowing whether people arrived at a decision through intuition or deliberation does not allow us to draw any universal conclusions about the normative correctness of their answer. ${ }^{14}$ Hence, premise (1) of the moral normativity inference is false. This implies that even if it were the case that utilitarian judgments in sacrificial dilemmas always result from System 2 processing (i.e., premise 2 of the moral normativity inference), the dual process framework does not allow us to draw any conclusion with respect to its normative status.

\section{On intuitive utilitarianism}

In the previous section, I argued against premise (1) of the moral normativity inference. Here I will review recent empirical evidence against premise (2). Assume for the sake of argument that the normative fallacy is correct. Any response that results from System 2 processing would be normatively correct. If the traditional dual process model of moral reasoning would be right - and utilitarian responses would typically result from System 2 processing - this would allow us to conclude that utilitarianism is normatively appropriate. The problem is that recent empirical evidence directly argues against the traditional characterization. ${ }^{15}$ For example, reasoners who give utilitarian responses to sacrificial dilemmas when they deliberate, typically give the same response when they are forced to reason purely intuitively. ${ }^{16}$ Hence, the response that is traditionally believed to require System 2 processing is often also cued by System 1.

Critical studies adopted the two-response paradigm. ${ }^{17}$ In this paradigm, participants are asked to give two consecutive responses to a moral dilemma. First, they need to answer as fast as possible with the first response that comes to mind. Afterward, they are given all the time they want to reflect on the problem and give a final response. To make sure that the response in the initial phase is indeed generated intuitively, participants need to answer within a stringent deadline and while their cognitive resources are burdened with a dual task (e.g., memorization of a complex visual pattern). These manipulations are known to "knock-out" deliberative thinking and help to guarantee that people do not reflect when they give their initial response. ${ }^{18}$ Results across multiple studies indicate that in the vast majority of cases, participants who gave a utilitarian final response (i.e., after deliberation was allowed), already gave this same response in the initial response phase. ${ }^{19}$ Hence, utilitarian responders do not need to deliberate to

\footnotetext{
${ }^{13}$ Stanovich, West (2000).

${ }^{14}$ Evans, Stanovich (2013).

${ }^{15}$ E.g. Baron (2017); Baron, Gürçay (2017); Bago, De Neys (2019); Białek, De Neys (2017); Gürçay, Baron (2017); Trémolière, Bonnefon (2014).

${ }^{16}$ E.g. Bago, De Neys (2019).

17 Thompson, Turner, Pennycook (2011).

${ }_{18}$ Bago, De Neys (2019).

${ }^{19}$ Bago, De Neys (2019).
} 
override an intuitively generated deontological answer; their initial hunch is already utilitarian in nature. ${ }^{20}$

I will refrain from discussing the implication of these findings for the dual process framework per se. ${ }^{21}$ The point I want to make here is that given that both utilitarian and deontological responses seem to be cued intuitively, there are no empirical grounds to feed the moral normativity inference. Even if System 2 would be normatively correct, the fact that both competing moral views are typically generated intuitively implies that there is no unique empirical mapping between types of reasoning and types of moral judgments.

\section{Conclusion}

In this commentary, I warned against a possible dual process misconception that could be used to (erroneously) infer that psychological research allows us to draw normative moral conclusions. I clarified the fact that the misconception is based on two erroneous premises. I argued that System 2 processing does not necessarily lead to normative correct decisions and that utilitarian moral judgments on sacrificial moral dilemmas do not necessarily require System 2. This cuts the ground from under the feet of the moral normativity inference and illustrates that it is indeed a fallacy. Although the dual process framework has great merits, it should not be used to make inferences about the normativity of moral judgments.

\section{Acknowledgments}

I would like to thank Jon Baron for his feedback on an earlier version of this manuscript.

\section{References}

Amer T., Campbell K.L., Hasher L. (2016), "Cognitive Control as a Double-edged Sword," Trends in Cognitive Sciences 20 (12): 905-915.

Bago B., De Neys W. (2017), "Fast logic?: Examining the Time Course Assumption of Dual Process Theory," Cognition 158: 90-109.

Bago B., De Neys W. (2019), “The Intuitive Greater Good: Testing the Corrective Dual Process Model of Moral Cognition," Journal of Experimental Psychology: General 148 (10): 1782-1801.

Bago B., De Neys W. (2019b), “The Smart System 1: Evidence for the Intuitive Nature of Correct Responding on the Bat-and-Ball Problem," Thinking \& Reasoning 25 (3): 257-299.

Baron J. (2017), “Utilitarian vs. Deontological Reasoning: Method, Results, and Theory,” [in:] Moral Inferences, J.-F. Bonnefon, B. Trémolière (eds.), Psychology Press, Hove: 137-151.

Baron J., Gürçay B. (2017), “A Meta-Analysis of Response-Time Tests of the Sequential Two-Systems Model of Moral Judgment," Memory and Cognition 45 (4): 566-575.

\footnotetext{
${ }^{20}$ For conceptually related findings on logical reasoning see De Neys (2017) and Bago, De Neys (2017), (2019b).

${ }^{21}$ But see De Neys (2012), (2017); De Neys, Pennycook (2019).
} 
Beilock S.L., DeCaro M.S (2007), “From Poor Performance to Success Under Stress: Working Memory, Strategy Selection, and Mathematical Problem Solving Under Pressure," Journal of Experimental Psychology: Learning, Memory, and Cognition 33 (6): 983-998.

Białek M., De Neys W. (2017), "Dual Processes and Moral Conflict: Evidence for Deontological Reasoners' Intuitive Utilitarian Sensitivity," Judgment and Decision Making 12 (2): 148-167.

De Neys W. (2012), "Bias and Conflict: A Case for Logical Intuitions," Perspectives on Psychological Science 7 (1): 28-38.

De Neys W. (2017), Dual Process Theory 2.0, Routledge, Oxon.

De Neys W., Pennycook G. (2019), "Logic, Fast and Slow: Advances in Dual-Process Theorizing," Current Directions in Psychological Science 28 (5): 503-509.

Evans J.S.B.T. (2002), “Logic and Human Reasoning: An Assessment of the Deduction Paradigm," Psychological Bulletin 128 (6): 978-996.

Evans J.S.B.T. (2011), “Dual-Process Theories of Reasoning: Contemporary Issues and Developmental Applications," Developmental Review 31 (2-3): 86-102.

Evans J.S.B.T., Stanovich K.E. (2013), “Dual-Process Theories of Higher Cognition: Advancing the Debate," Perspectives on Psychological Science 8 (3): 223-241.

Frankish K., Evans J.S.B.T. (2009), “The Duality of Mind: An Historical Perspective," [in:] In Two Minds: Dual Processes and Beyond, J. Evans, K. Frankish (eds.), Oxford University Press, Oxford: 1-29.

Greene J. (2013), Moral Tribes: Emotion, Reason and the Gap Between Us and Them, Penguin Press, New York.

Greene J., Haidt J. (2002), “How (and Where) Does Moral Judgment Work?,” Trends in Cognitive Sciences 6 (12): 517-523.

Gürçay B., Baron J. (2017), “Challenges for the Sequential Two-System Model of Moral Judgement," Thinking $\mathcal{E}$ Reasoning 23 (1): 49-80.

Kahneman D. (2011), Thinking, Fast and Slow, Farrar, Straus and Giroux, New York.

Stanovich K.E., West R.F. (2000), "Individual Differences in Reasoning: Implications for the Rationality Debate?," Behavioral and Brain Sciences 23 (5): 645-665.

Thompson V.A., Turner J.A.P., Pennycook G. (2011), "Intuition, Reason, and Metacognition," Cognitive Psychology 63 (3): 107-140.

Trémolière B., Bonnefon J.-F. (2014), “Efficient Kill-Save Ratios Ease up the Cognitive Demands on Counterintuitive Moral Utilitarianism," Personality and Social Psychology Bulletin 40 (7): 923-930. 